\title{
Some evidence for the management temporomandibular joint disorders
}

\author{
Abstracted from \\ List T, Axelsson S. \\ Management of TMD: evidence from systematic reviews and meta-analyses. \\ J Oral Rehabil 2010; 37: 430-451. \\ Address for correspondence: Thomas List, DDS, Odont. dr, \\ Department of Stomatognathic Physiology, Faculty of Odontology, \\ Malmo University, SE-20506 Malmo, Sweden. E-mail: Thomas.list@mah.se
}

\section{Question: What does systematic review evidence say about the management of temporomandibular joint disorders(TMD)?}

Data sources Medline (PubMed), the Cochrane Library, Bandolier and references in original articles and systematic reviews (SRs) were searched. Study selection Systematic reviews in English, Swedish or German that focused on the management of TMD were included. Reviews that covered oro-facial pain or TMD prevention were excluded.

Data extraction and synthesis Two investigators evaluated the methodological quality of each identified systematic review using two measurement tools, AMSTAR (http://www.biomedcentral.com/1471$2288 / 7 / 10$ ) and level of research design (LRD) score and the inter-rater reliability assess using Kappa statistics. Disagreements were resolved with discussion.

Results Thirty-eight systematic reviews met inclusion criteria and 30 were analysed ( 23 qualitative, seven meta-analyses). Ten of these reviews were related to occlusal appliances, occlusal adjustment or bruxism; eight to physical therapy; seven to pharmacologic treatment; four to TMJ and maxillofacial surgery; and six to behavioural therapy and multimodal treatment. The median AMSTAR score was 6 (range 2-11). Eighteen of the SRs were based on randomised clinical trials (RCTs), three were based on case-control studies and nine were a mix of RCTs and case series.

Conclusions There is some evidence that occlusal appliances, acupuncture, behavioural therapy, jaw exercises, postural training, and some pharmacological treatments can be effective in alleviating pain in patients with TMD. Evidence is insufficient for the effect of electrophysical modalities and surgery. Occlusal adjustment seems to have no effect according to the available evidence. One limitation of most of the SRs reviewed was that the considerable variation in methodology between the primary studies made definitive conclusions impossible.

\section{Commentary}

This very nicely written article has identified 38 published systematic reviews focused on the management of patients with Temporomandibular Disorders (TMD) and assessed their methodological quality by applying the criteria of the AMSTAR instru-

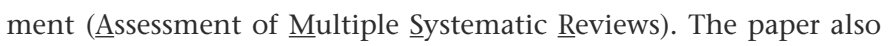
attempts to amalgamate the findings from these SRs to provide some evidence for best clinical management of patients with TMDs. It is praiseworthy that the authors warn that many of the SRs they reviewed concluded that it is impossible to suggest definitive conclusions due to considerable variation in methodology between the primary studies. The literature search was comprehensive and conducted in three bibliographic databases and the data selection and extraction were done in duplicate. A flow diagram of identified, screened, excluded and included studies was provided, which is in accordance with the guidance of the PRISMA statement (Preferred

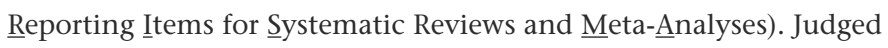
by comparing the identified SRs with the records of the EBD website of the American Dental Association (ebd.ada.org) it appears like their reference list was near complete with the exception of two SRs. ${ }^{1,2}$ The literature search cut-off date ended on September 2009. Subsequent to this date at least nine SRs have been published on the topics of TMDs and effectiveness of acupuncture $(n=4)$, surgery $(\mathrm{n}=2)$, and pharmacology $(\mathrm{n}=1)$. Additionally, Fricton and co-investigators have recently published two excellent SRs on TMDs and intra-oral appliances ${ }^{3}$ and the quality of RCTs focused on TMD management. ${ }^{4}$ Their intriguing conclusion is that much of the current evidence base for management of TMDs may be susceptible to systematic bias and that most past clinical studies should therefore be interpreted with caution. ${ }^{4}$ It is apparent that this investigator group is far more critical of the scientific quality of the included primary studies as a basis for formulating conclusions compared to many of the SR authors' conclusions as quoted and presented in the List and Axelsson paper.

Figure 2 is perhaps a bit perplexing for the reader and prone to misinterpretation. Several graphs show how many times primary studies were cited in one or more of the different systematic reviews within each treatment category, which may insinuate some form of selection bias of the primary studies. However, this is not necessarily the case since many of the SRs are very restrictive with regard to either diagnosis, eg, only anterior disc displacement with reduction, alternatively only reporting on the use of NTI splint type, alternatively limited to TMDs of myogenic and not arthrogenic pain origin and only treated by using a hard stabilisation splint. It is good 


\section{TEMPOROMANDIBULAR DISORDERS}

that the authors have not attempted to combine the findings of SRs, which appear to be similar on the surface, but once going into details are quite disparate. The reader should apply the same caution when interpreting the presented data.

Quality appraisal of SRs and primary studies aside, many of the interventions attempted over the years reflect underlying beliefs about aetiologies for TMDs and causes and effects of both interventions and no intervention. A clearer focus on a holistic approach to

\section{Practice points}

- Patients with temporomandibular disorders may benefit from a range of interventions managed either by the dental practitioner or by other health care professionals.

- Dentists can fabricate an occlusal appliance, while 'occlusal adjustment' has no documented benefits.

- Dentists and physicians can extend some forms of pharmacological treatment.

- Complementary or alternative health professionals can offer acupuncture or behavioural therapy.

- Physical therapists can provide postural training or jaw exercises.

- Electrophysical modalities and surgery are interventions that remain to be documented as effective for patients with TMDs. manage patients with TMDs is perhaps warranted. Clearly, we can expect differences in treatment outcomes amongst patients with acute pain versus chronic pain, high versus low levels of somatic complaints and anxiety ${ }^{5}$ and between the co-morbid versus otherwise healthy patients. Undersigned's position is that the management of patients with TMDs should be within a treatment team where the dentist's role is principally to rule out odontogenic causes for pain.

Asbjörn Jokstad

University of Toronto Faculty of Dentistry, Toronto, Canada

1. Tsukiyama Y, Baba K, Clark GT. An evidence-based assessment of occlusal adjustment as a treatment for temporomandibular disorders. J Prosthet Dent 2001; 86: 57-66. 2. Kreiner M, Betancor E, Clark GT. Occlusal stabilization appliances. Evidence of their efficacy. J Am Dent Assoc 2001; 132: 770-777.

3. Fricton J, Look JO, Wright E, et al. Systematic review and meta-analysis of randomized controlled trials evaluating intraoral orthopedic appliances for temporomandibular disorders. J Orofac Pain 2010; 24: 237-254.

4. Fricton JR, Ouyang W, Nixdorf DR, Schiffman EL, Velly AM, Look JO. Critical appraisal of methods used in randomized controlled trials of treatments for temporomandibular disorders. J Orofac Pain 2010; 24: 139-151.

5. Krogstad BS, Jokstad A, Dahl BL, Vassend O. The reporting of pain, somatic complaints, and anxiety in a group of patients with TMD before and 2 years after treatment: sex differences. J Orofac Pain 1996; 10: 263-269.

Evidence-Based Dentistry (2012) 13, 27-28. doi:10.1038/sj.ebd.6400848 